\title{
CALIDAD DE VIDA LABORAL EN TRABAJADORES DE SALUD PÚBLICA EN CHILE
}

QUALITY OF WORK LIFE IN PUBLIC HEALTH WORKERS IN CHILE

Recibido: 10 de Marzo del 2014 | Aceptado: 12 de Agosto del 2014

\author{
DANIEL PÉREZ-ZAPATA 1 ; REINALDO ZURITA2
}

(THE UNIVERSITY OF QUEENSLAND, Brisbane, Australia)

\section{RESUMEN}

Introducción: La Calidad de Vida Laboral es una variable psicosocial que incorpora factores intrínsecos, ligados a la experiencia subjetiva en el trabajo y factores objetivos, propios del contenido del trabajo y del ambiente laboral. Objetivos: Evaluar si la calidad de vida laboral está asociada a la satisfacción laboral y al síndrome de burnout y analizar si existen diferencias significativas en la evaluación de la calidad de vida laboral entre los estamentos organizacionales. Método: Bajo un diseño no experimental, analítico transversal, se recogieron datos de 72 trabajadores de un organismo de salud pública en Chile. Se utilizaron los cuestionarios de Satisfacción laboral de Melia et al. (1990) de Calidad de Vida laboral de Da Silva (2006) y el inventario de Maslach para evaluar Burnout (Maslach y Jackson, 1986). Resultados: La calidad de vida laboral se asoció significativamente con la satisfacción laboral y el síndrome de burnout. Además, se encontraron diferencias en función de la evaluación de calidad de vida laboral entre el estamento directivo y técnico $(p<0.048)$. Conclusión: Estos resultados sugieren que estas tres variables psicosociales estarían estrechamente vinculadas en contextos laborales ligados a la salud y que una comprensión general de la Calidad de Vida Laboral debería considerar un abordaje global.

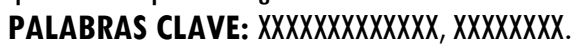

\section{ABSTRACT}

Introduction: The quality of work life is a relevant psychosocial variable, which has been studying in health organizations in recent years. This variable encompasses an intrinsic factor that links subjective experiences in work and an objective factor, which is associated with working content and working environment. Objectives: This study had two main aims. First, to determine whether quality of work life is correlated to job satisfaction and burnout syndrome; and second determine whether there is differences in terms of assessment of quality of work life in different organizational levels. Method: To achieve these goals, it collected 72 participants from a public health organization in Chile. Results: The quality of work life was significantly associated with job satisfaction and burnout syndrome. Additionally, it found that significant differences based on quality of work life's assessment between manager and technical levels $(p<0.048)$. Conclusion: These results suggest that these three psychosocial variables are closely related in health organizational contexts and a general comprehension of quality of work life should consider a global approach.

KEY WORDS: XXXXXXXXXXXXX, XXXXXXXX. 


\section{INTRODUCCIÓN}

La actividad laboral es una acción fundamental para el diario vivir y a la cual hay que destinar una gran cantidad de tiempo durante el ciclo de vida. Este hecho posiciona al trabajo como una variable primordial a la hora de comprender la calidad de vida de las personas (Sirgy, Reilly y Wu, 2008). En este marco, el concepto de Calidad de Vida Laboral (CVL) se ha convertido en una de las variables que progresivamente más estudios ha suscitado en el último tiempo, precisamente, por su carácter clave en el bienestar subjetivo $y$ en los resultados organizacionales (Da Silva, 2006) y/o porque contribuye en la comprensión de cómo los trabajadores de la salud perciben su entorno laboral y cómo se relaciona con otras importantes variables psicosociales al interior de las organizaciones, tales como satisfacción laboral o síndrome de burnout (Knox y Irving, 1997).

El camino que ha tenido que recorrer la investigación organizacional en CVL no ha sido sencillo. A pesar que en un comienzo los estudiosos poseían discrepancias con respecto a las variables que integraban este constructo, se pudo establecer cierto consenso sobre la identificación de dos perspectivas teórico-metodológicas elementales 0 básicas: la Calidad de Vida del Entorno Laboral y la Calidad de Vida Laboral Psicológica. La primera tiene como propósito suministrar una mejor calidad de vida a sus funcionarios mediante la consecución de objetivos organizacionales, enfocados en las condiciones de trabajo y en los elementos estructurales. Por su parte, la segunda perspectiva busca estudiar los factores asociados a la organización y a las relaciones interpersonales que determinan la experiencia del funcionario sobre su nivel de satisfacción, bienestar y salud. Del mismo modo, la CVL también puede ser entendida a partir de la distinción objetivo/subjetivo. Los elementos objetivos corresponden al sistema de gestión, a la organización interna del trabajo, horarios, contenido del puesto, salarios, etc.; mientras que los elementos subjetivos del trabajo son el grado de satisfacción laboral, los niveles de motivación, el reconocimiento del trabajo, el compañerismo, etc. (Da Silva, 2006). Es así que la CVL puede ser entendida como la experiencia subjetiva de los trabajadores que liga su implicación laboral con las funciones organizacionales que éstos desempeñan, las cuales están estrechamente vinculadas a sus niveles de satisfacción laboral, desempeño laboral y motivación (Segurado y Agulló, 2002).

Las implicaciones derivadas de los resultados empíricos, así como del análisis teórico de la CVL, sugieren que aquellas organizaciones que desarrollen un entorno de trabajo adecuado y que generen una percepción satisfactoria en sus empleados, producen una influencia positiva sobre la salud física, psicológica y emocional. Danna y Griffin (1999) realizaron una revisión de la literatura de salud y bienestar, en la cual hallaron una relación importante entre altos niveles de estas variables y CVL. Por otro lado, la CVL también repercute favorablemente en los resultados organizacionales (Chan y Wyatt, 2007; Da Silva, 2006; Gómez, 2010). Es más, existe evidencia que sugiere que los organismos que ofrecen condiciones de trabajo adecuadas, así como oportunidades de desarrollo profesional y personal para sus empleados, obtienen mayores ganancias y mejor consideración social (Lau y May, 1998; Harter, Schmidt y Keyes, 2002).

Los investigadores de CVL han propuesto, entre otras, dos variables psicosociales claves relacionadas con este concepto: la satisfacción laboral (SL) y el síndrome de Burnout (SB). Estas variables serían de capital importancia en la comprensión de las relaciones intra e inter personales en el lugar de trabajo. 
La SL ha sido estudiada ampliamente y se ha confirmado su significativa influencia en contextos organizacionales. Meliá et al. (1990) la conceptualizaron como una actitud o disposición para actuar de un modo determinado en relación a aspectos específicos del trabajo y la satisfacción con el mismo, que sería concebida como una actitud general resultante de muchas actitudes específicas asociadas a distintas aspectos o condiciones de trabajo. La SL no solamente es un proceso en el cual influyen factores de personalidad, sino también procesos psicosociales, en donde la persona realiza una interpretación y evaluación activa de las circunstancias de su ambiente laboral. Van Laar, Edwards y Easton (2007) encontraron que la SL fue el principal constructo que contribuyó a los resultados generales de CVL. Este resultado es concordante con conclusiones obtenidas por otros autores (Aketch, Odera, Chepkuto y Okaka, 2012; Baba y Jamal, 1991; Sirgy et al., 2001; Warr, Cook y Waal, 1979; Van Laar, Edwards y Easton, 2007).

El SB, también llamado síndrome de estar quemado, puede comprenderse como una respuesta prolongada a fuentes de estrés que se mantiene de forma crónica, tanto a nivel personal como a nivel laboral, determinados a partir del agotamiento emocional, despersonalización y cinismo e ineficacia profesional (Maslach, Shaufeli y Leiter, 2001). Sus principales componentes son tres: Agotamiento Emocional (AE), sentimientos de no poder dar más de sí a nivel emocional y a un deterioro de los propios recursos; Despersonalización (DP), respuesta de distancia interpersonal, conductas y emociones cínicas con respecto a los usuarios y/o compañeros de trabajo; y una baja Realización Personal (RP) referida a la disminución de los sentimientos de competencia y logro en el trabajo (Salanova y Llorens, 2008, 2009).

Una de las consecuencias negativas más importantes del SB es que el rendimiento profesional del sujeto disminuye significativamente, pudiendo repercutir en un mayor absentismo laboral y altas rotaciones. Asimismo, esta situación influye negativamente sobre la calidad del servicio entregado por parte de cualquier tipo de funcionario (Manzano, 2002), afectando directamente los niveles de bienestar de la población general.

Uno de los contextos laborales más complejos al interior de nuestra sociedad, y que a su vez, reúne uno de los abanicos más amplio de profesionales y no profesionales son las organizaciones de salud, donde el bienestar de sus funcionarios y los resultados que éstos sean capaces de lograr representa una función crítica para los miembros de una comunidad. Existe consenso en afirmar que los funcionarios de la salud se desempeñan habitualmente en entornos operacionales caracterizados por una elevada carga laboral, sumado a redes de apoyo social insuficientes (Lee, Dai, Park y McCreary, 2013). En un contexto como el descrito, los mecanismos de afrontamiento del estrés y de resolución de problemas que poseen, tanto funcionarios profesionales, como no profesionales resultan variados (GarcíaRamos, Luján-López y Martínez-Corona, 2007; Riveros, Berné y Múgica, 2010). Conocidos son los resultados que evidencian los altos niveles de estrés laboral del personal clínico (Murrels, Clinton y Robinson, 2005) y cómo éste repercute de forma adversa en su salud y bienestar general (Da Silva, 2006).

Los estudios de CVL en contextos de salud sugiere que los funcionarios experimentan una baja CVL percibida y un decaimiento de su motivación intrínseca (Khani, Jaafarpour y Dyrekuandmogadam, 2008; Hsu y Kernohan, 2006; Paredes y Paravic, 2006; Van Der Berg y Martins, 2013). En conjunto con estos resultados motivacionales, los aspectos objetivos del trabajo como el salario, la sobrecarga laboral y una carencia de tiempo influyen en el rendimiento cotidianos de los funcionarios 
de la salud en la percepción de CVL, afectando el despliegue y aplicación de sus habilidades profesionales en sus responsabilidades laborales (Murrels et al., 2005; Riveros et al., 2010; García-Ramos et al., 2007). Pérez-Zapata, PeraltaMontecinos y Fernández-Dávila (2014) proporcionaron evidencia que propone que la CVL percibida se encuentra positivamente asociada a satisfacción laboral, clima organizacional y engagement. En concordancia, dicho estudio, se expuso que el estamento técnico poseía una percepción más baja de CVL entre los estamentos comparados.

Otro aspecto que se ha destacado en el desarrollo del SB en profesionales de la salud, es que éste afecta la CVL de los funcionarios y funcionarias de la salud (GilMonte, Carretero y Roldán, 2005). Un estudio llevado a cabo en organizaciones de salud y de educación en México, encontró una relación significativa entre el desgaste emocional y la CVL, constatándose que los trabajadores de la salud eran más propensos a desarrollar niveles superiores de agotamiento emocional y de abandonar la organización en la cual se desempeñaban (Ortiz y Arias, 2009).

Si bien hay numerosos estudios que muestran que el SB afecta de forma importante a los funcionarios de la salud, hay una escasez de estudios que examinan la relación de CVL con SB en muestras latinoamericanas $\mathrm{y}$, especialmente, en trabajadores chilenos. En este contexto, los objetivos de este estudio fueron: (a) evaluar si existe relación entre la CVL y la SL y SB, y si es encontrada, en qué grado, y (b) analizar si existen diferencias en la percepción de CVL entre los distintos estamentos de una organización de salud pública en Chile.

\section{MÉTODO}

\section{Muestra}

Fueron recolectados datos de setenta y dos funcionarios de una organización pública de salud localizada en la ciudad de Arica, en Chile. En la Tabla 1 se describen las características socio demográficas de la muestra de funcionarios de salud.

TABLA 1.

Frecuencia y porcentajes de las variables edad, sexo y estamento.

\begin{tabular}{ccc}
\hline Edad & Frecuencia & Porcentaje \\
\hline 21 a 28 años & 14 & 19,4 \\
29 a 40 años & 29 & 40,3 \\
41 a 65 años & 29 & 40,3 \\
& & \\
\hline Sexo & Frecuencia & Porcentaje \\
\hline Femenino & 48 & 66,7 \\
Masculino & 24 & 33,3 \\
\hline Estamento & Frecuencia & Porcentaje \\
\hline Directivo & 6 & 8,3 \\
Profesional & 27 & 37,5 \\
Técnico & 28 & 38,9 \\
Administrativo & 11 & 15,3 \\
\hline
\end{tabular}

\section{Instrumentos}

Calidad de Vida Laboral. En el presente estudio se utilizó la escala elaborada por $\mathrm{Da}$ Silva (2006) en castellano, el cual tiene un formato de respuesta escala Likert que va desde uno ("Muy en Desacuerdo") a cinco ("Muy de Acuerdo"). Esta escala tiene 82 afirmaciones y está dividido de 11 dimensiones, las cuales son (a) Sistema de Gestión, (b) Relación con Compañeros, (c) Relación con Jefes, (d) Motivación, identificación y compromiso, Condiciones del entorno laboral, (f) Estrés y burnout, (g) Equilibrio trabajo-vida privada, (h) Satisfacción con el Rol y Potencial Motivador del Puesto, (i) Bienestar subjetivo derivado del trabajo, (j) Equidad Remunerativa y (k) Características de 
Personalidad. Cabe destacar que las puntuaciones de esta escala pueden ser utilizadas de forma multidimensional (a partir de la puntuación en cada una de las dimensiones descritas), 0 en forma unidimensional (a partir de la suma total de los reactivos). La confiabilidad del instrumento en el estudio de Da Silva fue de .9. En el presente estudio se obtuvo un alfa de Cronbach de .953, a partir de los 82 reactivos que componen la escala.

Satisfacción Laboral. Para medir SL, se aplicó el cuestionario de satisfacción S21/26 de Meliá et al. (1990), en cual se encuentra en castellano. El instrumento consta de 26 reactivos estructurado en torno a 6 dimensiones, (a) Satisfacción con la supervisión y participación en la organización, (b) Satisfacción con las remuneraciones y prestaciones, (c) Satisfacción Intrínseca, (d) Satisfacción con el Ambiente Físico, (e) Satisfacción con la Cantidad de Producción y (f) Satisfacción con la calidad de producción. La escala de valoración del instrumento va desde uno ("Muy Insatisfecho") a cuatro ("Muy Satisfecho"0); siendo posible trabajar, tanto con los puntajes de cada dimensión, como con la puntuación total al sumar las mismas. La fiabilidad del instrumento es de .897. En el presente estudio se obtuvo un alfa de Cronbach de .897. Los resultados pueden oscilar entre los 0 a 104 puntos, donde un mayor puntaje se traduciría en una mejor evaluación de la SL.

Síndrome de Burnout. Se utilizó la versión en castellano del Maslach Burnout Inventory (Maslach y Jackson, 1986), el cual posee 22 afirmaciones y se compone de tres dimensiones: Agotamiento Emocional, Despersonalización y Realización Personal. Estas dimensiones se responden de acuerdo a una escala que va desde cero ("Nunca") a seis ("Siempre"). En el presente estudio se obtuvo un alfa de Cronbach de 0,84 .

\section{Procedimientos}

Se utilizó un diseño no experimental analítico transversal. A cada uno de los participantes se le entregó un cuadernillo, en el cual se encontraban las escalas de CVL, SL y SB. Además, se solicitó información sociodemográfica relativa al sexo, rango etario y estamento laboral. En términos generales, los participantes tardaron alrededor de una hora en contestar el cuadernillo. En relación a los aspectos éticos, la aplicación de los instrumentos fue previamente informada a los responsables de los centros de salud pública correspondientes y se informó oportunamente que los cuestionarios eran anónimos, que podían ser contestados libremente y que podían dejarlos sin completar si es que se consideraba que éstos afectaban su privacidad.

Con respecto al análisis de datos empleado, éste se llevó a cabo en el paquete estadístico SPSS IBM versión 21, en cual se realizaron análisis descriptivos, pruebas de análisis de varianza (ANOVA) de una vía, pruebas post-hoc de Bonferroni y correlaciones.

\section{RESULTADOS}

Al analizar la diferencia de medias en la CVL (Tabla 2), no se encontró resultados estadísticamente significativos para las variables edad $(p>, 366)$ y sexo $(p>, 544)$. Al analizar las diferencias en la media entre los diversos estamentos (directivos, profesionales, técnicos y administrativos), esta fue estadísticamente significativa, $F$ $(3,62)=2,851, p<.044, \eta^{2}=.148$. Las diferencias se encontraron entre el estamento directivo y técnico $(p<.048)$, experimentando los primeros mayores índices de CVL que los últimos. Adicionalmente, y a modo descriptivo, se incluyen las medias y desviaciones estándar de las otras variables presentes en el estudio. 
TABLA 2.

Media Aritmética y desviación estándar ( $\overline{\boldsymbol{X}}$; s) para las variables Calidad de Vida Laboral, Satisfacción Laboral y Síndrome de Burnout (Agotamiento Emocional, Despersonalización, Realización Personal) separado por edad, sexo y estamento.

\begin{tabular}{lllllll}
\hline & & CVL & SL & AE - MBI & DE - MBI & RP - MBI \\
\hline Edad & 21 a 28 años & 67,$5 ; 2,9$ & 68,$5 ; 13,7$ & 17,$4 ; 10,8$ & 5,$3 ; 4,9$ & 35,$9 ; 12,3$ \\
& 29 a 40 años & 65,$2 ; 2,2$ & 67,$7 ; 11,5$ & 20,$4 ; 11,9$ & 7,$6 ; 5,5$ & 40,$6 ; 9,1$ \\
\multirow{4}{*}{ Sexo } & 41 a 65 años & 67,$7 ; 2,3$ & 67,$6 ; 12,4$ & 20,$0 ; 13,6$ & 4,$5 ; 4,4$ & 40,$6 ; 8,3$ \\
& Femenino & 71,$6 ; 2,8$ & 68,$3 ; 12,3$ & 19,$3 ; 12,8$ & 5,$6 ; 5,2$ & 40,$3 ; 10,5$ \\
& Masculino & 66,$0 ; 2,1$ & 67,$5 ; 12,2$ & 20,$3 ; 11,4$ & 6,$4 ; 4,9$ & 38,$5 ; 7,4$ \\
& Directivo & 75,$7 ; 15,6$ & 74,$2 ; 11,12$ & 24,$0 ; 12,2$ & 2,$0 ; 6,0$ & 46,$0 ; 4,4$ \\
& Profesional & 68,$4 ; 9,2$ & 69,$3 ; 12,9$ & 19,$8 ; 12,6$ & 4,$9 ; 5,0$ & 41,$1 ; 9,2$ \\
& Técnico & 63,$3 ; 9,8$ & 66,$3 ; 10,9$ & 18,$1 ; 12,6$ & 6,$6 ; 5,1$ & 39,$1 ; 8,4$ \\
& Administrativo & 66,$5 ; 7,9$ & 65,$1 ; 14,2$ & 19,$8 ; 12,3$ & 6,$9 ; 5,1$ & 34,$6 ; 13,6$ \\
\hline
\end{tabular}

Al analizar la relación de la CVL con las variables independientes, se encuentra que la SL tiene una relación positiva y significativa con CVL $(r=.710, p<.001)$; mientras que el SB obtuvo una correlación negativa y estadísticamente significativa con CVL $(r=-.419, p<.001)$.

TABLA 3.

Correlaciones generales de Calidad de Vida Laboral con Satisfacción Laboral, Agotamiento Emocional, Despersonalización y Realización Personal.

\begin{tabular}{lc}
\hline & CVL \\
\hline Satisfacción Laboral &, $710^{* *}$ \\
SB - Agotamiento Emocional &,$- 314^{* *}$ \\
SB - Despersonalización &,$- 379^{* *}$ \\
SB - Realización Personal &, $252^{*}$ \\
\hline${ }^{* *}$ La correlación es significativa al nivel 0,01 (bilateral). \\
$\quad$ * La correlación es significativa al nivel 0,05 (bilateral).
\end{tabular}

\section{DISCUSIÓN}

El presente estudio tuvo por objetivo determinar si la satisfacción laboral y el síndrome de burnout estaban asociados con la calidad de vida laboral y si había diferencias entre estamentos en las evaluaciones de Calidad de Vida Laboral en una organización pública de salud en Chile. Después de realizar los análisis de datos correspondientes, se constató que la Satisfacción Laboral está positiva y fuertemente asociada al concepto de Calidad de Vida Laboral. Este resultado es consistente con las conclusiones de numerosos estudios (Aketch et al., 2012; Baba y Jamal, 1991; Pérez-Zapata et al., 2014; Sirgy et al., 2001; Warr et al., 1979; Van Laar et al., 2007), los cuales afirman que el nivel de satisfacción que experimentan los trabajadores en su entorno laboral es uno de los principales componentes de la calidad de vida en el trabajo. Además de este resultado entre estas dos variables psicosociales, se encontró que la relación entre el síndrome de burnout y calidad de vida laboral resultó significativa, evidenciando una dirección directa con la Realización Personal, e inversa con el Agotamiento Emocional y la Despersonalización en esta muestra particular. Estos indicadores son concordantes con los resultados encontrados en organizaciones de salud y educacionales (Ortiz y Arias, 2009). Por tanto, este estudio logra proporcionar evidencia adicional que confirma las relaciones arriba mencionadas en una organización pública de salud en Chile. En lo referente a la existencia de diferencias entre los grupos comparados según estamentos, se pudo apreciar que el técnico obtuvo una percepción de CVL más baja que el estamento directivo. 
Estos resultados pueden ser explicados desde diferentes puntos de vista. En uno de ellos, se asume que en los servicios de atención primaria de salud tienen la función de responder a las necesidades emergentes de la ciudadanía de una forma rápida y oportuna. La prestación de estos servicios depende de los funcionarios e insumos de salud disponibles para dar respuestas a estas necesidades. Uno de los hechos que rodean a la salud pública en Latinoamérica, es que en muchas ocasiones los requerimientos de la población no pueden ser satisfechos con los recursos físicos y humanos existentes. Esta situación puede producir una sobrecarga laboral y niveles de estrés importantes en los funcionarios de salud lo que afectaría la calidad de vida laboral percibida y otras variables psicosociales relevantes en los contextos organizacionales, como por ejemplo, la Satisfacción Laboral y el Síndrome de Burnout. Da Silva (2006) ha propuesto que estos dos factores son vitales para entender la Calidad de Vida Laboral en la mayoría de las organizaciones. El hecho que el estamento técnico posea niveles más bajos de CVL, podría ser explicado por estos elementos. Además, hay que tener en cuenta que el estamento técnico de los servicios de salud está constituido en su gran mayoría por técnicos paramédicos, quienes, en la mayoría de las veces, son los primeros en interactuar con los usuarios de salud, brindando un importante servicio clínico. Desde una perspectiva intuitiva, el hecho que los trabajadores estén satisfechos con un rango significativo de sus aspectos laborales y que perciban niveles bajos de agotamiento emocional y despersonalización, debería contribuir a aumentar los niveles de Calidad de Vida Laboral percibida.

Otro importante factor que podría explicar estas relaciones es el grado de satisfacción que poseen los trabajadores de la salud con sus recompensas monetarias y sociales. El hecho que los trabajadores estén relativamente conformes con su remuneración y con las oportunidades de ascenso laboral, además de los diferentes modos de reconocimiento social presente en los trabajos, debería tener un efecto en la CVL. Además de este factor, otro aspecto importante que puede explicar en especial el resultado de diferencia en la percepción de CVL entre estamentos, es la justicia laboral percibida. La percepción que los funcionarios de una organización determinada sean tratados más o menos del mismo modo, sin importar su posición en el organigrama y la cantidad de remuneración obtenida, es primordial para construir una percepción laboral positiva. De lo contrario, los funcionarios pueden desarrollar niveles afectivos negativos contra distintos aspectos de la organización, desde compañeros de trabajo a funcionarios que estén ocupando posiciones de jefatura. De esta manera, queda pendiente para próximos estudios analizar la relación de estas variables con Calidad de Vida Laboral, resultando relevante la exploración más detallada entre los factores de los instrumentos utilizados, además de incrementar el tamaño de la muestra para facilitar el correcto ajuste de los resultados. 
REFERENCIAS

Aketch, J. R., Odera, O., Chepkuto, P., y Okaka, O. (2012). Effects of quality of work life on job performance: theoretical perspectives and literature review. Current Research Journal of Social Sciences, 4(5), 383-388.

Baba, V. V., y Jamal, M. (1991). Routinisation of job context and job content as related to employees quality of working life: a study of psychiatric nurses. Journal of Organizational Behavior, 12, 379-386.

Chan, K. W., y Wyatt, T. A. (2007). Quality of Work Life: A Study of Employees in Shangahi, China. Asia Pacific Business Review, 13(4), 501-507.

Da Silva, M. (2006). Nuevas perspectivas de la Calidad de Vida Laboral y sus relaciones con la eficacia organizacional. Tesis Doctoral, Universidad de Barcelona, España.

Danna, K., y Griffin, R. W. (1999). Health and well-being in the workplace: A review and synthesis of the literature. Journal of Management, 25, 357-384.

García-Ramos, M., Luján-López, M. E., y Martínez-Corona, M. A. (2007). Satisfacción Laboral del Personal de Salud. Revista de Enfermería del Instituto de Seguro Social, 15 (2), 63-72.

Gil-Monte, P. R., Carretero, N., y Roldán, M. D. (2005). Algunos procesos psicosociales sobre el síndrome de quemarse por el trabajo (burnout) en profesionales de enfermería. Ansiedad y Estrés, 11, 281290.

Gómez, M. A. (2010). Calidad de Vida Laboral en empleados temporales del valle de Aburrá-Colombia. Revista Ciencias Estratégicas, 18(24), 225-236.

Harter, J. K., Schmidt, F. L., y Hayes, T. L. (2002). Business unit-level relationship between employee satisfaction, employee Engagement, and business outcomes: A meta-analysis. Journal of Applied Psychology, 87 (2), 268-279.

Hsu, M.,Y., y Kernohan, G. (2006). Dimensions of hospital nurses' quality working life. Journal of Advanced Nursing, 54, 120-131.

Khani, A., Jaafarpour, M., y Dyrekuandmogadam, A. (2008). Quality of Nursing Work Life. Journal of Clinical and Diagnostic Research, 2, 1169-1174.

Knox, S., y Irving, J. A. (1997). An interactive quality of work life model applied to organisational transition. Journal of Nursing Administration, 27(1), 39-47.

Lau, R., y May, B. (1998) A Win- Win Paradigm for Quality of Work life and Bussiness Performance. Human Resource Developpment Quarterly, 9(3), 211- 227.

Lee, Y., Dai, Y., Park, C., y McCreary, L. L. (2013). Predicting quality of work life on nurses' intention to leave. Journal of Nursing Scholarship, 45(2), 160-168.

Manzano, G. (2002). Burnout y Engagement en un colectivo preprofesional estudiantes universitarios. Boletín de Psicología, 74, 79-102.

Maslach, C., Schaufeli, W. B., y Leiter, M. P. (2001). Burnout. Annual Review of Psychology, 52, 397-422.

Maslach, C. \& Jackson, S.E. (1986). Maslach Burnout Inventory: Second Edition. Palo Alto, CA: Consulting Psychologists Press.

Meliá, J. L., Pradilla, J. F., Martí, N., Sancerni, M. D., Oliver, A., y Tomás, J. M. (1990). Estructura Factorial, Fiabilidad y Validez del cuestionario de satisfacción 21/26: un instrumento con formato dicotómico orientado al trabajo profesional. Revista de Psicologia Universitas Tarraconensis, 12(1/2), 25-39.

Murrels, T., Clinton, M., y Robinson, S. (2005). Job satisfaction in nursing: validation of a new instrument for the UK. Journal of Nursing Management, 13, 296-311.

Ortiz, J. A., y Arias, F. (2009). El desgaste profesional (burnout) y calidad de vida laboral como predictores de la búsqueda de otro trabajo en profesionales de la salud y de la educación en el occidente de México. Ciencia y Trabajo, 34, 222-226.

Paredes, L. A., y Paravic, T. (2006). Percepción de violencia de usuarios hospitalizados en los servicios clínicos de un hospital público de la Región de la Araucanía de Chile. Ciencia y Enfermería, 12 (1), 39-51.

Pérez-Zapata, D., Peralta-Montecinos, J., y Fernández-Dávila, P. (2014). Influencia de variables organizacionales en la Calidad de Vida Laboral de funcionarios del sector público de salud en el extremo norte de Chile. Universitas Psychologica,

Riveros, J., Berné, C., y Múgica, J. M. (2010). Gestión y satisfacción en servicios de salud de Chile: contraste entre las percepciones de los funcionarios y los usuarios. Revista Médica de Chile, 138, 630-638. 
Salanova, M. y Llorens, S. (2008). Estado actual $y$ retos futuros en el estudio del burnout. Papeles del Psicólogo, 29(1), 59-67.

Salanova, M. y Llorens, S. (2009). Exposición a la Tecnología de la Información y la Comunicación y su Relación con el Engagement. Ciencia y Trabajo, 32, 55-62.

Segurado, A. S. \& Agulló, E. (2002). Calidad de vida laboral: hacia un enfoque integrador desde la psicología social. Psicothema, 14, 828-836.

Sirgy, M. J., Efraty, D., Siegel, P., y Lee, D. (2001). A new measure of quality-of-work life (QWL) based on need satisfaction and spillover theories. Social Indicators Research, 55, 241-302.

Sirgy, M. J., Reilly, N. P., y Wu, J. (2008). A work-life identity model of well-being: towards a research agenda linking qualityof-work-life (QWL) programs with quality of life (QQL). Applied Research Quality Life, 3, 181-202.

Van Der Berg, Y., y Martins, N. (2013). The relationship between organisational trust and quality of work life. SA Journal of Human Resource Management/SA Tydskrif vir Menslikehulpbronbestuur, 11(1), Art.\#392, 13 pages. http://dx.doi.org/10.4102/sajhrm.v11i1.392

Van Laar, D. L., Edwards, J. A. \& Easton, S. (2007). The Work-Related Quality of Life (QoWL) scale for Healthcare Workers, Journal of Advanced Nursing. 60 (3), 325-333.

Warr P., Cook J., y Wall T. (1979) Scales for the measurement of some work attitudes and aspects of psychological well being. Journal of Occupational Psychology, 52, 129-148. 